\title{
SPECTRUM AGGREGATION SCHEME IN A WIRELESS BROADBAND DATA TRANSCEIVER SYSTEM
}

\author{
Da Guo, ${ }^{*}$ Yong Zhang,, Guangnian $\mathrm{Xu},{ }^{* *}$ and Park Hyeongchun ${ }^{* * *}$
}

\begin{abstract}
This paper investigates the design of a wireless broadband data transceiver (WBDT) system. The WBDT system works on hundreds of narrowband discrete channels. Cognitive radio (CR) technology is adopted to detect idle channels. Discrete channels are aggregated into large frequency bands to provide higher transmission capability than traditional wireless data transceivers (WDTs). Guard gaps are used to avoid interference among WBDTs/WDTs working on adjacent channels according to existing WDT standards. To make full use of the idle channels, a new spectrum aggregation (SA) method, maximum space first assignment (MSFA), is proposed after analysing the performance of the WBDT system. MSFA provides a way to reduce the negative effect of the guard gap. The simulation and analysis results show that the novel system architecture and MSFA improve spectral efficiency.
\end{abstract}

\section{Key Words}

Cognitive radio, spectrum aggregation, data transceiver system

\section{Introduction}

\subsection{Background and Motivation}

Wireless data transceivers (WDTs) are widely employed in industry, the military, weather forecasting, and other areas. WDTs can work at different frequency bands, such as $230 \mathrm{MHz}, 433 \mathrm{MHz}, 868 \mathrm{MHz}$, and $2.4 \mathrm{GHz}$ typically. According to China government regulations published in 1991, the State Grid Corporation of China, the China Meteorological Administration, and other government ministries have been granted a license for part of the frequency

* School of Electronic Engineering, Beijing University of Posts and Telecommunications, Beijing, P.R.C; e-mail: \{guoda, yongzhang\}@bupt.edu.cn

** Research center for technology, The State Grid Zhejiang electric power company, Hangzhou, Zhejiang Province, P.R.C; e-mail: xu_guangnian@zj.sgcc.com.cn

*** Chinese department, Soon Chun Hyang University, ASAN, South Korea; e-mail: hyeongchun@126.com

Corresponding author: Da Guo

Recommended by Dr. Xiaonv Hu

(DOI: 10.2316/Journal.206.2018.5.206-0068) from 223.025 MHz to $235 \mathrm{MHz}$. Currently, WDT manufacturers must obey the Generic Specification for Data Transceiver standard (GB/T 16611-1996) [1] published in 1996 in China. There are some similar standards in other countries such as ARIB STD-T67 and ETSI-EN 300-220.

The WDT frequencies can be used by eight licensed private networks and some unlicensed amateur stations in China. As shown in Fig. 1, the white space between $223.025 \mathrm{MHz}$ and $235 \mathrm{MHz}$ is used for amateur stations (unlicensed channels), and the lines indicate the licensed channels that are used by the eight private networks. The bandwidth of a channel is $25 \mathrm{kHz}$ and each WDT provides 9.6-kbps communication capability. The private network users cannot use the licensed channels of other private networks. Furthermore, Fig. 1 shows that the channels owned by private network operators are discontinuous. Obviously, the WDT's communication capability cannot meet the needs of the intelligent power grid.

A novel wireless broadband data transceiver (WBDT) system was employed in the power wireless private network [2]-[5]. The WBDT system was developed based on TD-LTE technology and works on $230 \mathrm{MHz}$. The WBDT system has wider coverage than the LTE system due to its lower frequency. According to the experimental results in the trial network in Haiyan, Zhejiang Province, China, the base station can cover a $10-\mathrm{km}$ radius in the town area, and only three WBDT base stations are required to satisfy the power communication requirements of the whole town (shown in Fig. 2) [2].

To use the unlicensed channels and unused licensed channels, we propose a novel WBDT architecture. The main features of this architecture include two aspects: Cognitive radio (CR) capability to sense idle channels and spectrum aggregation (SA) capability to aggregate the discrete channels to provide broad bandwidth. The SA method is the emphasis in our work. The novel WBDT system can be employed for all amateur stations and all the licensed private network operators.

\subsection{Related Work}

Our proposed system uses orthogonal frequency division multiplex (OFDM) technology because OFDM can provide 


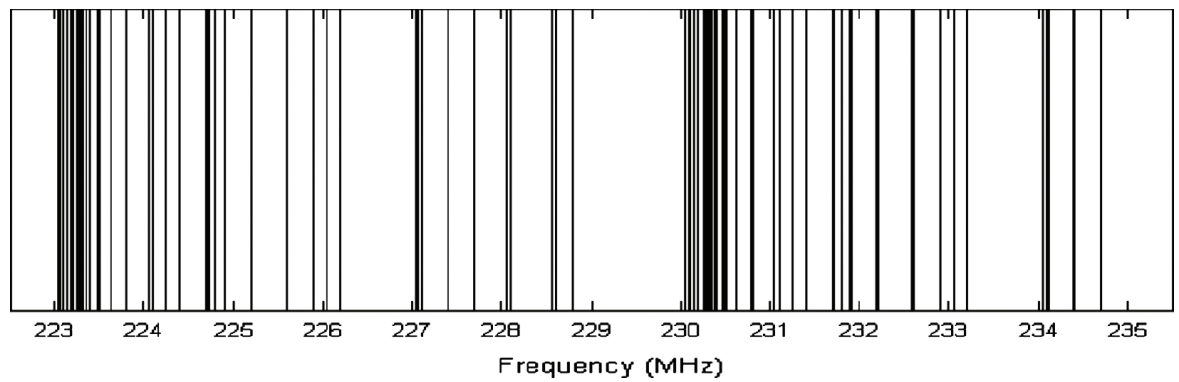

Figure 1. WDT frequency spectrum.

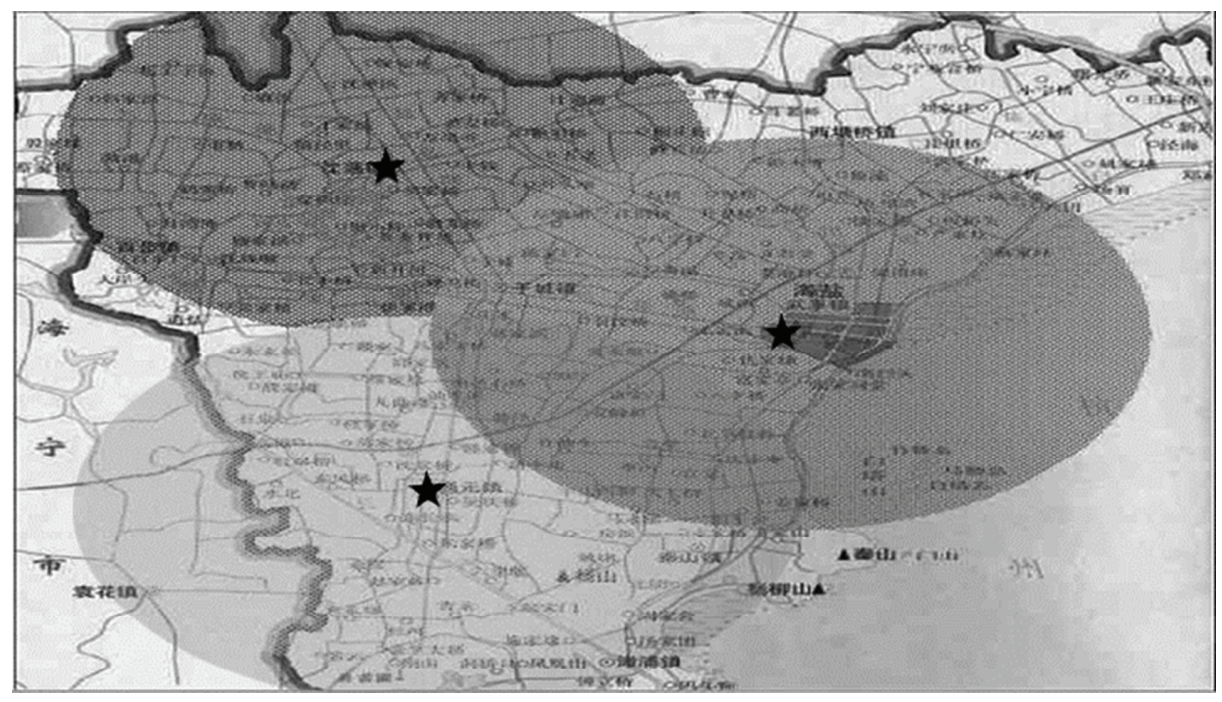

Figure 2. Power wireless private network in Haiyan, Zhejiang Province.

high spectrum efficiency. Some researchers have tried to implement OFDM technology in discontinuous frequency bands. Poston proposed a solution called discontinuous OFDM (DOFDM), which enables a single user to access several spectrum fragments using only one radio front in idle TV channels [6]. DOFDM provides a good reference design for the WBDT system.

CR technology can sense the radio environment, obtain information about idle spectral, and enhance communication capability. As mentioned above, some licensed private network operators and amateur stations seldom use their channels because of the low communication capability of traditional WDTs. This provides a perfect application environment for CR technology in the $230 \mathrm{MHz}$ spectrum. Currently, many researchers are focusing their interest on CR, including spectrum sensing [7], spectrum handoff [8], and spectrum access [9]. The IEEE 802.22 workgroup is also developing standards for CR in TV broadcast service. This paper does not give a detailed discussion of CR implementation in a WBDT system because our application is similar to existing research.

SA technology can extend the bandwidth in LTE systems to achieve high data rate transmission [10]. SA is one of the key features of the LTE-advanced system and has been investigated by many researchers because it is difficult to find enough contiguous spectrum bands in LTE systems [11]-[13]. Most investigations of carrier aggregation focus on the application in LTE systems [14]-[17]. Some resource scheduling and management schemes have been proposed for LTE systems in the last several years [18]-[22]. A special simulation platform was developed for a cognitive LTE system [23], and SA is an important technology in the $5 \mathrm{G}$ mobile communication system [24].

However, the frequency feature of WBDT systems is different from that of LTE and 5G systems. The spectrum is illustrated in Fig. 3. First, there are more than 400 channels, far more than in LTE systems. Generally, several discrete spectrum bands are aggregated to provide broadband communication in LTE systems. Second, the channel spacing is too small, and data transmission is carried on several channels. The guard gap bandwidth between adjacent channels is significant in the whole frequency band. Thus, the guard gap utilization plays a key role in the channel assignment solution. These differences create great challenges. Some researchers have proposed solutions involving spectrum assignment [25], but they all ignore the influence of the guard gap.

Cao et al. [26] and Cao et al. [27] estimate the system capacity using carrier aggregation in the licensed channels owned by the State Grid Corporation of China for a power private network. All 40 channels that belong to the State Grid Corporation of China are aggregated to form a $1-\mathrm{MHz}$ spectrum band and provide more than $2.4 \mathrm{Mbps}$ communication capability, but the scheme only works in one private network and cannot use the idle channels that belong to amateur stations and other private network 


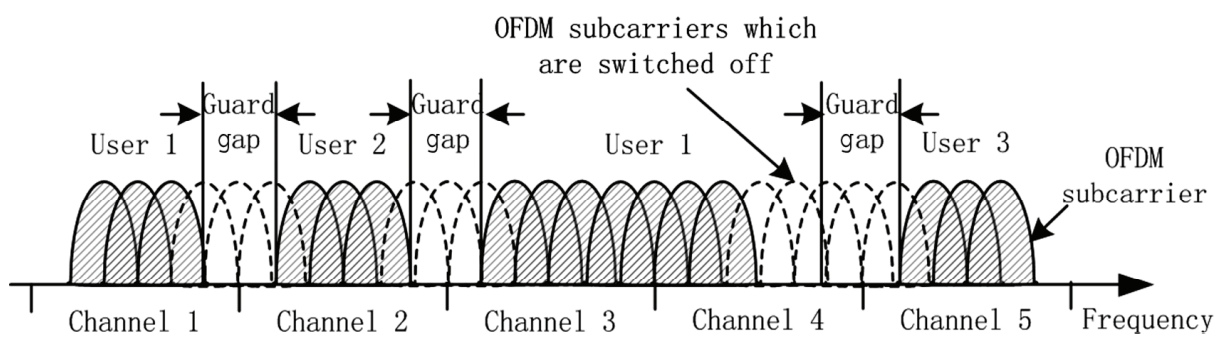

Figure 3. Channels, guard gaps, and subcarriers.

operators. Yidong et al. [28] proposes a spectrum-sharing algorithm and power allocation method based on WDBTs, and Cao et al. [29] propose a two-stage double-threshold local spectrum sensing algorithm to improve detection performance and reduce computing complexity.

To improve the performance of WBDT systems, we focus our research on a SA solution to provide higher data rate transmission. In this paper, we formulate the SA problem in WBDT systems and propose a novel spectrum assignment method: the maximum space first assignment (MSFA) algorithm. The simulation and analysis show that this solution promotes spectrum efficiency.

\section{System Model and Problem Formulation}

\subsection{Spectrum Feature}

CR technology is adopted in our proposed system to detect idle frequencies because few people use traditional WDTs in cities due to the low data rate. This means that private network operators can use their licensed frequencies as well as frequencies of amateur stations. Amateur stations can also use more than one channel to extend their bandwidth for higher data rates using one radio front at a time.

Before stating the problem, we define some terms:

Channel: the channels shown in Fig. 3 are used by traditional WDTs and can carry several OFDM subcarriers. The traditional WDTs can only work on one channel at a time. In our proposal, several channels can be aggregated to serve as a pair of communication nodes. According to the China standard, the channel width is $25 \mathrm{kHz}$.

Guard Gap: guard gaps are used to avoid interference among WDTs working on adjacent channels. The guard gap bandwidth should adhere to the existing traditional WDT standard. The guard gap can be omitted if the continuous channels are assigned to the same user. According to the China standard, the bandwidth of the guard gap is $9 \mathrm{kHz}$. OFDM subcarriers that overlay the guard gaps should be switched off.

User: the user is one communication terminal whose physical layer adopts OFDM technology. One user may occupy one channel or several channels, but one channel can be occupied by only one user.

Spectrum fragment: the spectrum fragment assigned to a user is composed of the continuous available channels. For example, User 1 owns two spectrum fragments including Channels 1, 3, and 4 in Fig. 3.

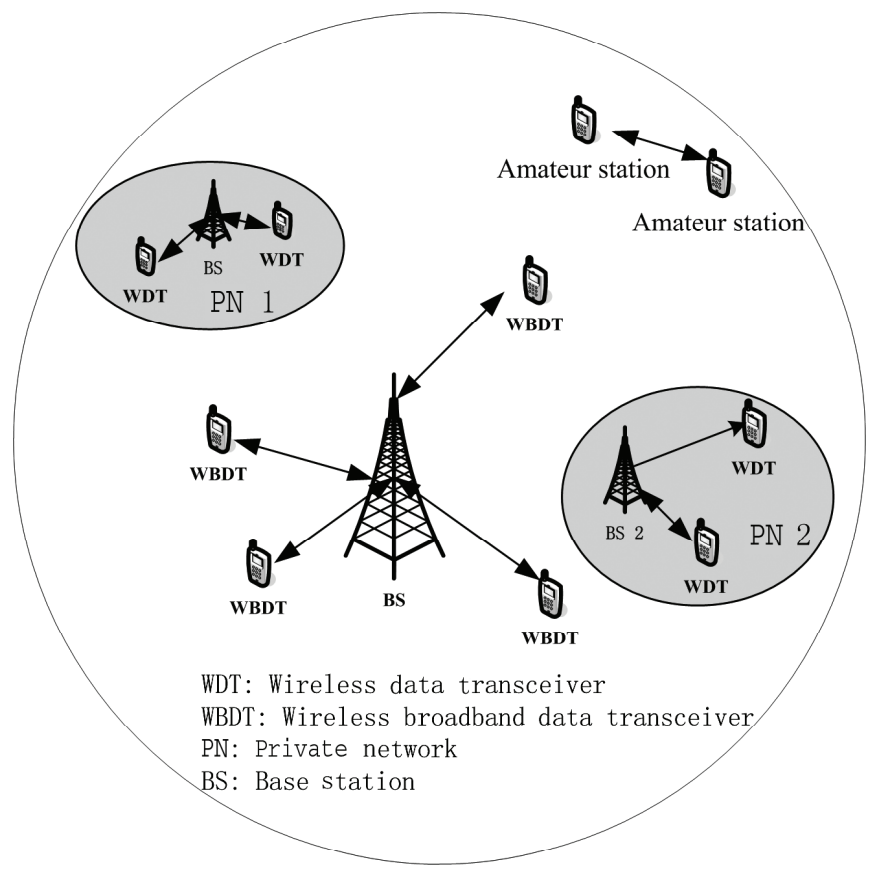

Figure 4. Network architecture.

\subsection{Problem Definition}

The network adopts the cellular architecture shown in Fig. 4. Some private networks work with other private networks and amateur stations. WBDT and WDTs are fixed stations that communicate with their corresponding base stations. WBDT and WDTs may belong to different operators. For purposes of analysis, we propose the following assumptions:

- WBDTs with CR capability can sense the entire $12-\mathrm{MHz}$ frequency band and learn frequency usage information.

- The filter adopted in the proposed WBDT system is perfect enough that DOFDM can switch the corresponding subcarriers on or off normally and interference between the adjacent channels does not exist. In fact, the guard gaps prevent this interference.

As we know, the spectrum of WDTs/WBDTs is from $223.025 \mathrm{MHz}$ to $235 \mathrm{MHz}$. The diversity of path loss between $223.025 \mathrm{MHz}$ and $235 \mathrm{MHz}$ is $0.77 \mathrm{~dB}$ according to the following HATA model [30]:

$$
\begin{aligned}
L= & 46.3+33.9 \log f_{c}-13.82 \log h_{t}-a\left(h_{r}\right) \\
& +\left(44.9-6.55 \log h_{t}\right) \log d,
\end{aligned}
$$


where $L$ is the path loss in $\mathrm{dB}, f_{c}$ is the working frequency in $\mathrm{MHz}, h_{t}$ is the height of the transmitting antenna in meters, $h_{r}$ is the height of the receiving antenna in meters, and $d$ is the distance from the transmitter to the receiver in kilometres.

Suppose that the signal-to-noise ratio (SNR) of user $i$ at channel $j$ is $\alpha_{i, j} S N R_{i, 1} . \alpha_{i, j}$ is the SNR diversity factor at the difference channel. $S N R_{i, 1}$ is the reference SNR for user $i$ at channel $1(223.025 \mathrm{MHz})$.

The entire $12-\mathrm{MHz}$ frequency band is divided into $N$ channels. There are $M$ users in a cellular network. The channel width is $W_{c} \mathrm{KHz}$, the guard gap bandwidth is $W_{\text {gap }} \mathrm{KHz}$, and the subcarrier bandwidth is $W_{s c} \mathrm{KHz}$. User $i$ demands $D_{i}$ kbps, so the data rate of user $i$ using channel $j$ is

$$
R_{i, j}=W_{s c} \log _{2}\left(1+\Gamma * \alpha_{i, j} \mathrm{SNR}_{i, j}\right) \quad(\mathrm{kbps})
$$

where $\Gamma=-1.5 / \ln \left(5 B_{k}^{\min }\right)$ is the $\mathrm{SNR}$ gap related to a minimal targeted bit-error-ratio $B_{k}^{\min }$. According to the existing regulation, the number of WDT channels is 440, so there are few diversity factors among difference channels. For simplicity, let $\alpha_{i, j}=1$. This means the SNR of user $i$ is approximately identical. Then, we rewrite the data rate of user $i$ as follows:

$$
R_{i}=W_{s c} \log _{2}\left(1+\Gamma * S N R_{i, 1}\right) \quad(\mathrm{kbps}) .
$$

Suppose the channel characteristic is known for all nodes. The channel state matrix is given by

$$
S=\left[\begin{array}{cccc}
s_{1,1} & s_{1,2} & \cdots & s_{1, N} \\
s_{2,1} & s_{2,2} & \cdots & s_{2, N} \\
\vdots & \vdots & \ddots & \vdots \\
s_{M, 1} & s_{M, 2} & \cdots & s_{M, N}
\end{array}\right], \quad s_{i, j} \in\{0,1\}
$$

where $s_{i, j}=0$ denotes that channel $j$ is available for user $i$ and $s_{i, j}=1$ denotes that channel $j$ is occupied by existing service and cannot be used in channel assignment.

The binary channel assignment matrix is

$$
A=\left[\begin{array}{cccc}
a_{1,1} & a_{1,2} & \cdots & a_{1, N} \\
a_{2,1} & a_{2,2} & \cdots & a_{2, N} \\
\vdots & \vdots & \ddots & \vdots \\
a_{M, 1} & a_{M, 2} & \cdots & a_{M, N}
\end{array}\right], a_{i, j} \in\{0,1\}, a_{i, j} s_{i, j} \neq 1
$$

which denotes whether user $i$ occupies channel $j$. If $a_{i, j}=1$, channel $j$ is assigned to user $i$. Otherwise, it is not.

The channel is occupied by only one pair of users, thus

$$
\sum_{i=1}^{M} a_{i, j} \leq 1, \quad \forall j=1,2, \ldots N
$$

The number of assigned spectrum fragments can be derived from the channel assignment matrix. Assume the assigned frequency is made up of $k_{i}$ spectrum fragments. The maximum available capacity for user $i$ under the above channel assignment matrix is

$$
C_{i}=R_{i} \times \text { floor }\left[\left(W_{c} \times \sum_{j=1}^{N} a_{i, j}-k_{i} \times W_{\text {gap }}\right) / W_{s c}\right] \text {. }
$$

The maximum available capacity per user is not less than the requirement of user $i$.

$$
D_{i} \leq C_{i}=R_{i} \times \text { floor }\left[\left(W_{c} \times \sum_{j=1}^{N} a_{i, j}-k_{i} \times W_{\text {gap }}\right) / W_{s c}\right]
$$

We evaluate the performance of the spectrum assignment strategies by their spectrum efficiency. The basic idea to optimize the spectrum assignment strategy is to maintain the network capability. If the transmission request occupies a different number of channels using different spectrum assignment strategies, the fewer the number of channels that are occupied, the better the strategy is. In other words, a better strategy can gain higher spectral efficiency. Thus, to minimize the number of occupied channels, we rewrite the above equations as:

$$
\begin{aligned}
& \left(a_{i, j}, k_{i}\right)=\arg \min \sum_{i=1}^{M} \sum_{j=1}^{N} a_{i, j} \\
& \text { s.t. } C_{i} \geq D_{i} \\
& \sum_{i=1}^{M} a_{i, j} \leq 1, \quad \forall j=1,2, \ldots N, \quad a_{i, j} \in\{0,1\} \\
& a_{i, j} s_{i, j} \neq 1
\end{aligned}
$$

It is difficult to obtain a globally optimum solution. In fact, the solution is not unique, but we derive it as follows from (5)

$$
\begin{gathered}
D_{i} \leq C_{i}=R_{i} \times \text { floor }\left[\left(W_{c} \times \sum_{j=1}^{N} a_{i, j}-k_{i} \times W_{\text {gap }}\right) / W_{s c}\right] \\
\leq R_{i} \times\left(W_{c} \times \sum_{j=1}^{N} a_{i, j}-k_{i} \times W_{\text {gap }}\right) / W_{s c} \\
\sum_{j=1}^{N} a_{i, j} \geq\left(D_{i} \times W_{s c} / R_{i}+k_{i} \times W_{\text {gap }}\right) / W_{c} .
\end{gathered}
$$

\subsection{Complexity Analysis}

According to the China government regulation published in 1991 [1], there are 440 channels used by WDTs in the spectrum from $223.025 \mathrm{MHz}$ to $235 \mathrm{MHz}$. Within that spectrum, 100 channels are owned by licensed private networks 
and 380 channels are owned by unlicensed amateur stations. The number of available idle channels is $N \in[1,440]$. Binary channel assignment matrix $A$ has $M \times N$ elements. $A$ may have $2^{M \times N}$ states. Even though some of these states are not feasible, the exhaustive search algorithm that is used to find the optimal solution is infeasible when there are so many narrowband discrete channels.

To reduce the complexity of implementation, the original optimum problem can be simplified as follows:

$$
\left(a_{i, j}, k_{i}\right)=\arg \min \sum_{j=1}^{N} a_{i, j}, \quad \forall i=1,2, \ldots M
$$

As a matter of fact, the communication requests that arrive at the WBDTs and base stations should queue up and wait before being scheduled. The channel state matrix will be updated by the base stations if the channel characteristic changes due to transmission of other private networks or amateur stations, but the base station still deals with new arrivals individually from the queue.

From (10) and (11), we can obtain a suboptimal solution by reducing the number of assigned spectrum fragments $k_{i}$, so we propose a novel method called MSFA to obtain a suboptimum solution.

\section{MSFA Algorithm}

Essentially, MSFA is a greedy algorithm. Assume that the base stations know the channel characteristics and communication requirements of each WBDT. Generally, the assigned channels of one user will be divided into more spectrum fragments if the user needs more subcarriers. First, the request for maximum channels is assigned to the longest continuously idle channels. While the networks are receiving transmission requests from $M$ users at the same time, the MSFA algorithm should work as follows:

(1) users obtain frequency usage information of the $N$ channels, including idle/busy status, the SNR from the user to the corresponding base station, etc.

(2) According to the frequency usage information, establish the channel state matrix $S=\left\{S_{i, j}\right\}$.

(3) Calculate the number of channels demanded by user $i$ as follows:

$$
\begin{aligned}
F_{i}= & \operatorname{ceil}\left(\left(D_{i} \times W_{s c} / R_{i}+k_{i} \times W_{\text {gap }}\right) / W_{c}\right) \\
& \left(i \in[1, \mathrm{M}], k_{i}=1\right)
\end{aligned}
$$

assume that user $\bar{i}$ has the largest demand. $F_{\bar{i}}=\operatorname{Max}\left(F_{i}\right)$.

(4) Determine the sizes of the available spaces $F S_{k}(k \geq 1)$. Any available space must be made up of continuously idle channels. The available space having the maximum continuous channels is denoted as the largest available space $\operatorname{Max}\left(F S_{k}\right)$.

It should be noted that the new assignment process for one user should not start unless the previous user completes its assignment process as follows.

(5) The largest available space $\left(\operatorname{Max}\left(F S_{k}\right)\right)$ carries the largest demand $\left(F_{\bar{i}}\right)$. (a) If $\operatorname{Max}\left(F S_{k}\right) \geq F_{\bar{i}}$, user $\bar{i}$ employs $F S_{k}$, marks the corresponding channels with $\bar{i}$ in $\left\{S_{i, j}\right\}$, sets $F_{\bar{i}}=0$, and recalculates $F S_{k}$.

(b) If $\operatorname{Max}\left(F S_{k}\right)<F_{\bar{i}}$, user $\bar{i}$ employs $F S_{k}$, marks the corresponding channels with $\bar{i}$ in $\left\{S_{i, j}\right\}$, and sets $F S_{k}=0$. Recalculate the number of demanded channels $F_{\bar{i}}$.

$$
F_{\bar{i}}=F_{\bar{i}}-\text { floor }\left(\left(\operatorname{Max}\left(F S_{k}\right) \times W_{c}-W_{\text {gap }}\right) / W_{s c}\right)
$$

(6) Update $F_{\bar{i}}$ and $F S_{k}$. Go to Step 5 and make the largest available space, $\operatorname{Max}\left(F S_{k}\right)$, carry the remaining demand of $\bar{i}\left(F_{\bar{i}}\right)$ until $F_{\bar{i}}=0$ or $\sum_{k} F S_{k}=0$.

For user $\bar{i}$, when the transmission request cannot be satisfied, the second largest request $F_{l}\left(F_{l}<F_{\bar{i}}\right)$ is attempted until all of the users are traversed. If $\sum_{i=1}^{M} F_{i}=0$, it means that all the requests are satisfied.

(7) Update $F_{i}$ and $F S_{k}$ and go to Step 5 until $\sum_{k} F S_{k}=0$ or $\sum_{i=1}^{M} F_{i}=0$.

If $\sum_{k} F S_{k}=0$ and $\sum_{i=1}^{M} F_{i} \neq 0$ after all of the users are traversed, it means that the current network cannot carry more unassigned services.

The network should constantly maintain the channel state matrix $S=\left\{S_{i, j}\right\}$. If a new request arrives from user $i$ while the network is working, the MSFA algorithm could work as follows:

(a) Count the current available spaces $F S_{k}(k \geq 1)$ that are made up of continuously idle channels.

(b) Calculate the number of demanded channels $F_{i}$ $(i \in[1, \mathrm{M}])$ using $(12)$.

(c) The largest available space $\left(\operatorname{Max}\left(F S_{k}\right)\right)$ carries the request from user $i\left(F_{i}\right)$.

If $\operatorname{Max}\left(F S_{k}\right) \geq F_{i}$, user $i$ employs $F S_{k}$, marks the corresponding channels with $i$ in $\left\{S_{i, j}\right\}$, and recalculates $F S_{k}$.

If $\operatorname{Max}\left(F S_{k}\right)<F_{i}$, user $i$ employs $F S_{k}$, marks the corresponding channels with $i$ in $\left\{S_{i, j}\right\}$, and sets $F S_{k}=0$. Recalculate the number of demanded channels $F_{i}$ using (13).

(8) Update $F_{i}$ and $F S_{k}$ and go to Step 3 until $\sum_{k} F S_{k}=0$ or $F_{i}=0$.

Herein, we do not re-sort all $F_{i}$ and reassign the channels, because the channel handover will lead to excessive signalling and delay costs.

The MSFA algorithm runs in the base station and schedules all the available channels. The base station determines the spectrum assignment and notifies the mobile node of this information. 
Table 1

Simulation Parameters

\begin{tabular}{|l|l|}
\hline Simulation Parameter & Value \\
\hline Request of user $i$ & $50 \mathrm{kbps} \leq D_{i} \leq 100 \mathrm{kbps}$ \\
\hline SNR of user $i$ & $10 \mathrm{db} \leq \mathrm{SNR}_{i} \leq 20 \mathrm{db}$ \\
\hline Width of channel & $W_{c}=25 \mathrm{kHz}$ \\
\hline Width of subcarrier & $2 \mathrm{kHz} \leq W_{s c} \leq 10 \mathrm{kHz}$ \\
\hline Width of guard gap & $1 \mathrm{kHz} \leq W_{\text {gap }} \leq 15 \mathrm{kHz}$ \\
\hline $\begin{array}{l}\text { Minimal targeted } \\
\text { bit-error-ratio }\end{array}$ & $B_{k}^{\mathrm{min}}=0.0001$ \\
\hline
\end{tabular}

\section{Simulation and Evaluation}

In this section, we will evaluate the MSFA algorithm by computer simulation and compare its performance with the contiguous assignment (CA) algorithm. In the CA algorithm, the resources are sequentially allocated to users according to the order of the demands in the queue. Because no existing assignment algorithms are proposed currently, the CA may be a simple solution.

Before the computer simulation, we evaluated the complexity of the CA and MSFA algorithms. In the CA algorithm, the main time consumption is the marking process. The base station should mark $O\left(\sum_{i=1}^{M} D_{i}\right)$ channels as $\left(\sum_{i=1}^{M} D_{i} \leq N\right)$. In the MSFA algorithm, the main time consumption includes the marking and sort processes. $\quad k_{i}\left(k_{i} \leq N / 2\right)$ available spaces and $M$ users should be sorted. The time complexity is related to sorting algorithms. For example, the time complexity is $O\left(N^{2}\right)+O\left(M^{2}\right)$ if a bubble sort algorithm is adopted.

The simulation scenario is shown in Fig. 4. There are some existing users including amateur stations and private networks in the proposed system's coverage. The existing users already occupy some channels. Assume that the total spectrum is divided into $N(N=440)$ channels. The probability that a channel is idle is $p(0<p<1)$, and the number of users in the proposed system is $M(1 \leq M \leq 15)$. The channels are randomly assigned to existing users according to the probability $p$ in our simulation program.

The transmission request and SNR of a user are random values. The detailed values of the simulation parameters are listed in Table 1. We repeated our simulation 100 times and obtained the average values.

In the WDBT system, the channel width and number of channels are constant. The adjustable parameters include the number of users $M$, channel idle probability $p$, users' data rate $D_{i}$, and received $\mathrm{SNR}_{i}$. Because the values of $M, p, D_{i}$, and $S N R_{i}$ describe the system busy status, we set $D_{i}$ as a random value from $50 \mathrm{kbps}$ to $100 \mathrm{kbps}$ and compared the spectral efficiency under different values of $\mathrm{SNR}_{i}$ and $p$. Here, $i$ denotes the user ID.

First, we compared the spectrum efficiency in different idle channel probabilities. From Fig. 5, we can see that the MSFA algorithm has better spectrum efficiency than CA under different SNRs. The spectrum efficiency gain of the MSFA algorithm is more than $20 \%$ higher than that

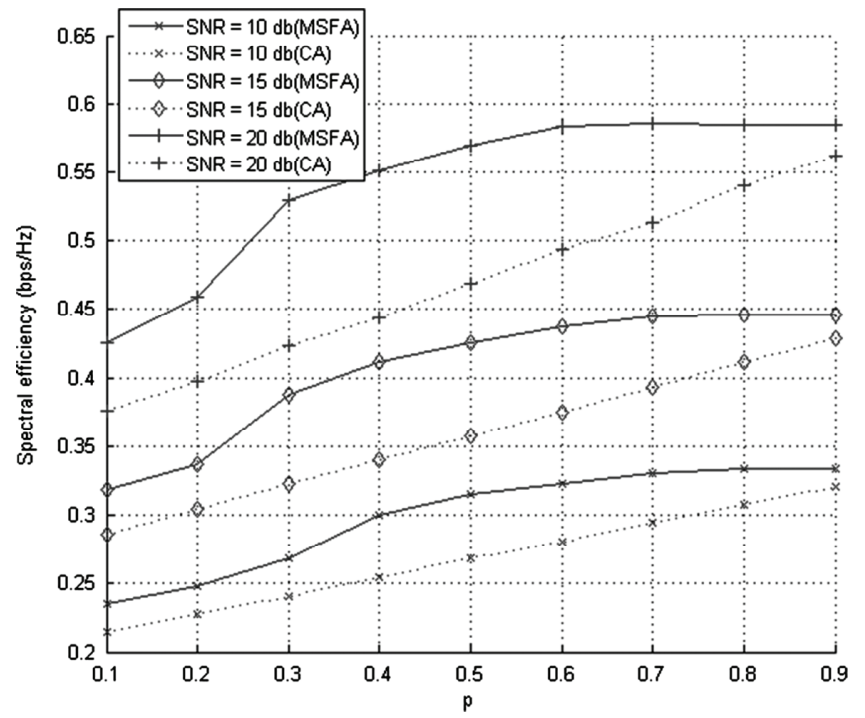

Figure 5. Spectrum efficiency $\left(M=10, \quad W_{s c}=5 \mathrm{kHz}\right.$, $\left.W_{\text {gap }}=9 \mathrm{kHz}\right)$.

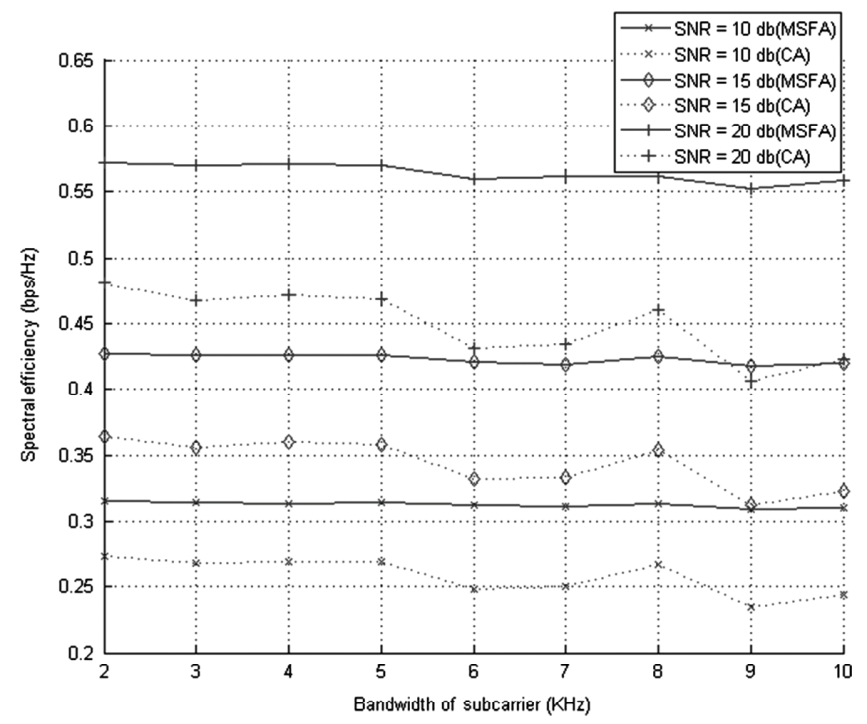

Figure 6. Spectrum efficiency $\left(M=10, \quad W_{\text {gap }}=9 \mathrm{kHz}\right.$, $p=0.5)$.

of the CA algorithm when the idle channels probability is in the middle status. When the spectrum is not too busy, there are more contiguous channels and the effect of aggregation decreases. In addition, when the idle channel probability increases, the effect of aggregation under discrete channels decreases. Furthermore, the spectrum efficiency of the MSFA and CA algorithm will increase when the frequencies become idle.

We compared the effect of the different bandwidths of the subcarrier and SNR. The subcarrier bandwidth changes from $2 \mathrm{kbps}$ to $10 \mathrm{kbps}$. Fig. 6 shows that the performance of the MSFA algorithm is very stable. The spectrum efficiency of the MSFA is higher than that of the CA algorithm. Generally, the spectrum efficiency of the CA algorithm decreased slightly, whereas the bandwidth of the subcarrier increased. But, the point of $W_{s c}=8 \mathrm{kHz}$ is an exception because the frequency band for subcarriers is $16 \mathrm{kHz}$ and can carry only 2 subcarriers. The guard gap can be taken full advantage of when $W_{s c}=8 \mathrm{kHz}$. 


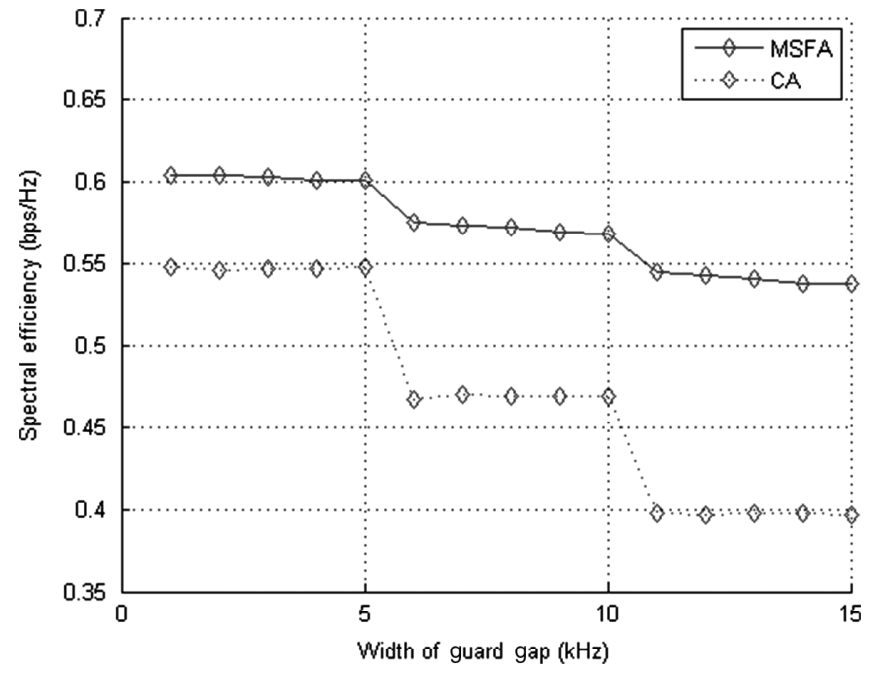

Figure 7. Spectrum efficiency $\left(M=10, \quad W_{s c}=5 \mathrm{kHz}\right.$, $p=0.5)$.

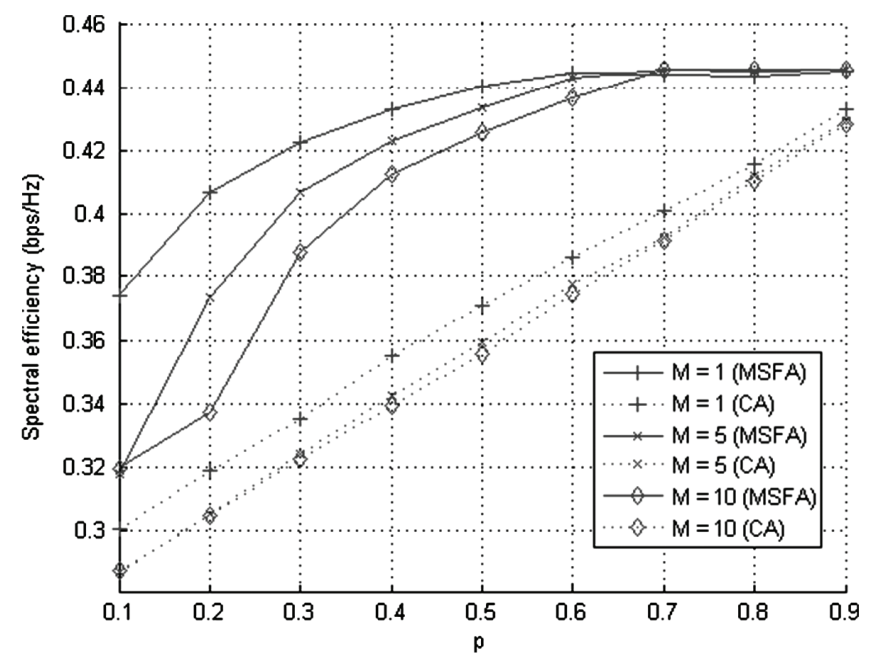

Figure 8. Spectrum efficiency $\left(\mathrm{SNR}=15 \mathrm{db}, W_{s c}=5 \mathrm{kHz}\right.$, $\left.W_{\text {gap }}=9 \mathrm{kHz}\right)$.

Even though the width of the guard gap is standardized, we tried to change the value of $W_{\text {gap }}$ and investigate its effect on spectrum efficiency. The performance of the MSFA and CA algorithms were analysed under different guard gap bandwidths. From Fig. 7, we see that the smaller the guard gap is, the higher the spectral efficiency is. The reason is that the smaller guard gap can reduce spectrum waste. However, a smaller guard gap means that better filters have to be employed in the design of proposed WBDT systems. In addition, because the bandwidth of a subcarrier is $5 \mathrm{kHz}$, the lines decrease in a series of cascades.

Finally, we studied the performance with different numbers of users. The trend of spectrum efficiency with different numbers of users in Fig. 8 is similar to that in Fig. 5. The guard gap has to be reserved between adjacent users. The channel may not be made full use of by users. $M=1$ especially indicates that users arrive one by one, which means that MSFA can work well with different numbers of users.

It should be noted that the spectrum efficiency for 5 users $(M=5)$ is only slightly higher than that for 10 users $(M=10)$ when $p=0.1$ in Fig. 8. The average spectrum efficiency is 0.3168 for 10 users and 0.3190 for 5 users.
The reason is that the most of the channels are busy and there is not enough available space for users when $p=0.1$. Furthermore, from Figs. 5-8, we find that a higher SNR will achieve higher spectrum efficiency.

\section{Conclusion}

Traditional WDTs cannot satisfy the market demand. We propose a novel WBDT architecture to solve this problem. We put great emphasis on the SA algorithm, and the theoretical analysis and algorithm process are presented. The performance of the MSFA algorithm is discussed with different parameters, and the simulation results show that the architecture and MFSA can achieve better spectrum efficiency than a $\mathrm{CA}$ algorithm.

It should be noted that the MSFA algorithm is designed for maximum spectrum efficiency. This may lead to unfairness among services when the requests in the queue are scheduled. Quality of service (QoS) guarantees is an important issue in wireless communication systems, and such guarantees in WDBT systems will be discussed in the future.

Although the WBDT design incorporated here is specific to China, the methodology and findings should be relevant to a global audience and offer a complementary perspective.

\section{Acknowledgement}

This work is supported by the national Major Science and Technology Special Projects (No. 2014ZX03004002). The authors would like to thank the reviewers for their detailed reviews and constructive comments, which have helped improve the quality of this paper.

\section{References}

[1] The state bureau of quality and technical supervision of China, "Generic specification for data transceiver", GB/T 16611-1996, 1996.

[2] J. Cao, J. Liu, S. Zhu, and Y. Zhang, A wide coverage wireless communication scheme for the intelligent distribution network, 2012 World Congress on Information and Communication Technologies (WICT), Trivandrum, India, 2012, 999-1003.

[3] J. Cao, L. Jianming, Y. Zhang, X. Li, and L. Zeng, Developing a power wireless private network based on TD-LTE Technology for Intelligent Distribution Networks, International Conference on Automatic Control and Artificial Intelligence (ACAI 2012), Xiamen, China, 2012, 890-893.

[4] L.X. Yang, K.A. Hou, C. Gong, et al. The application of TD-LTE230M wireless power broadband network technology in smart power-stealing prevention system, Applied Mechanics and Materials, 738, 2015, 1217-1220.

[5] Y.Sun, X.N. Lin, and L.M. Chen. Utilizing indicator computing assistant to facilitate distribution automation in LTE wireless private network, Applied Mechanics and Materials, 687, 2014, 3239-3242.

[6] J.D. Poston and W. D. Horne, Discontiguous OFDM considerations for dynamic spectrum access in idle TV channels, In Proc. IEEE Int. Symp. New Frontiers Dynamic Spectrum Access Networks, Baltimore, MD, USA, November 2005, vol. 1, 607-610.

[7] Y. Tevfik and A. Huseyin, A survey of spectrum sensing algorithms for cognitive radio applications, IEEE Communications Surveys \& Tutorials, 11(1), 2010, 116-128.

[8] Y. Zhang, Spectrum handoff in cognitive radio networks: Opportunistic and negotiated situations, In Proc. 2009 IEEE 
International Conf. on Communications, Dresden, Germany, Jun. 2009, IEEE Press, 3510-3515.

[9] A. Garhwal and P.P. Bhattacharya, A survey on dynamic spectrum access techniques for cognitive radio, International Journal of Next-Generation Networks, 3(4), 2012,15-32.

[10] Y. Kakishima, T. Kawamura, Y. Kishiyama, and H. Taoka, Experimental evaluation on throughput performance of asymmetric carrier aggregation in LTE-advanced, In Proc. IEEE Vehicular Technology Conference (VTC Spring), Budapest, Hungary, 2011, IEEE 73rd.

[11] 3GPP, Summary of LTE advanced requirements presented at the workshop, REV-080058, 3GPP Specification. Available at http://www.3gpp.org/ftp/workshop/2008-0407 RAN_IMT_Advanced/Docs/REV-080058.zip

[12] M. Iwamura, K. Etemad, M.-H. Fong, R. Nory, and R. Love, Carrier aggregation framework in 3GPP LTE-advanced, IEEE Communications Magazine, 48(8), August, 2010, 60-67.

[13] Z. Shen, A. Papasakellariou, J. Montojo, D. Gerstenberger, and F. Xu, Overview of 3GPP LTE-advanced carrier aggregation for $4 \mathrm{G}$ wireless communications, IEEE Communications Magazine, 50(2), 2012, 122-130

[14] L. Chen, W. Chen, X. Zhang, and D. Yang, Analysis and simulation for spectrum aggregation in LTE-advanced system, Proc. Vehicular Technology Conference Fall (VTC 2009-Fall), 2009 IEEE 70th, Anchorage, Alaska, USA, 20-23 Sept. 2009, 1-6.

[15] L. Liu, M. Li, J. Zhou, X. She, L. Chen, Y. Sagae, and M. Iwamura, Component carrier management for carrier aggregation in LTE-advanced system, Proc. IEEE Vehicular Technology Conference (VTC Spring), 2011 IEEE 73rd, Budapest, Hungary, 15-18 May 2011, 1-6.

[16] H. Wang, C. Rosa, , and K. Pedersen, Uplink component carrier selection for LTE-advanced systems with carrier aggregation, 2011 IEEE International Conference on Proc. Communications (ICC), Kyoto, Japan, 5-9 June 2011, 1-5.

[17] N.J. Diego, B. Gerhard, S. Abdallah, et al., Interference protection mechanism for LTE-advanced radio access networks supporting dynamic spectrum access, 2013 IEEE Wireless Communications and Networking Conf. (WCNC), Shanghai, China, 7-10 April 2013, 3603-3608.

[18] G.D. Ntouni, A.A.A. Boulogeorgos, D.S. Karas et al. Interband carrier aggregation in heterogeneous networks: Design and assessment, 2014 11th International Symposium on. IEEE Wireless Communications Systems (ISWCS), Barcelona, Spain, 2014, 842-847.

[19] Y. Zhang, Y. Zhang, Y. Teng, et al. An efficient carrier scheduling scheme in cognitive LTE-Advanced system with carrier aggregation, 2014 IEEE 25th Annual International Symposium on. IEEE Personal, Indoor, and Mobile Radio Communication (PIMRC), Washington, DC, 2014.

[20] K. Zheng, F. Liu, W. Xiang, et al. Dynamic downlink aggregation carrier scheduling scheme for wireless networks, IET Communications, 8(1), 2014, 114-123.

[21] H. Shajaiah, A. Abdelhadi, and C. Clancy, A price selective centralized algorithm for resource allocation with carrier aggregation in LTE cellular networks, Wireless Communications and Networking Conference (WCNC), 2015 IEEE. IEEE, 2015.

[22] H. Shajaiah, A. Abdel-Hadi, and C. Clancy, Utility proportional fairness resource allocation with carrier aggregation in 4G-LTE, Military Communications Conference, MILCOM 2013 - 2013 IEEE. IEEE, 2013, 412-417.

[23] Y. Zhang, Y. Fang, M. Song, et al., System level simulation platform for Cognitive LTE, Journal of Supercomputing, 72(7), 2015, 2623-2634.

[24] S. Rostami, K. Arshad, and P. Rapajic, A joint resource allocation and link adaptation algorithm with carrier aggregation for 5G LTE-Advanced network, Telecommunications (ICT), 2015 22nd International Conference on. IEEE, Sydney, NSW, Australia, 2015, 102-106.

[25] W. Wang, Z. Zhang, A. Huang, Spectrum aggregation: Overview and challenges, Network Protocols and Algorithms, 2(1), 2010, 184-196.

[26] J. Cao, J. Liu, S. Zhu, and D. Gao, Application and performance analysis of carrier aggregation for a novel power wireless private network, 2012 World Congress on Information and Communication Technologies (WICT), Dalian, China, 2012, 1004-1008.

[27] J. Cao, J. Liu, X. Li, et al., Performance analysis of a new power wireless private network in intelligent distribution networks, Power Engineering and Automation Conference (PEAM), 2012 IEEE. IEEE, 2012, 1-4.

[28] Y. Yidong, Z. Dongyan, W. Guangyu, A study of spectrum sharing technology based on $230 \mathrm{MHz}$ electric private wireless network, Application of Electronic Technique, 41(8), 2015 79-82.

[29] J. Cao and J. Liu, A two-stage double-threshold local spectrum sensing algorithm research for the power private communication network, Proceedings of the CSEE, 35(10), 2015, 2471-2479.

[30] T.S. Rappaport, Wireless Communications Principles and Practice, 2nd ed., (Electronics Industry, 2006), New Jersey, $105-154$.

\section{Biographies}
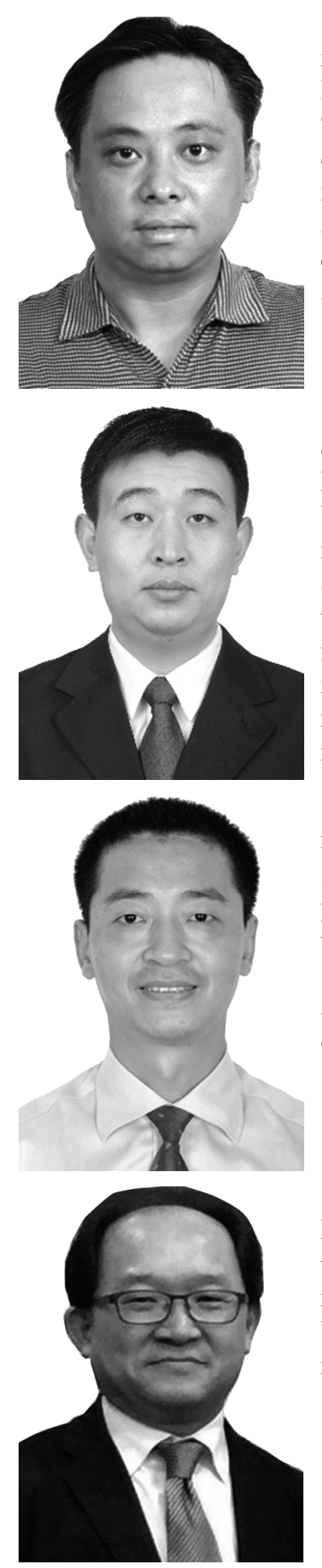

Da Guo received his Ph.D. degree in Electrical Engineering from Beijing University of Posts and Telecommunications, and he is currently a senior engineer at that institution. His research interests are in mobile communications, opportunistic networks, WSN, and P2P networks.

Yong Zhang has been an associate professor in the School of Electronic Engineering at Beijing University of Posts and Telecommunications, PR China. He holds a Ph.D. degree from Beijing University of Posts and Telecommunications. His research interests include self-organizing networks, mobile communications, and cognitive networks.

Guangnian $X u$ is a senior engineer working for the State Grid Zhejiang Electric Power Company, China. He graduated from the HoHai University in August 1993. He engages in the research and application of power system communications technology.

Park Hyeongchun received his Ph.D. degree from Shandong University, PR China. Currently he is a professor at Soon Chun Hyang University. His research interest is in linguistics. 